\title{
A method to compensate for incomplete 24-hour urine collections in nutritional epidemiology studies
}

\author{
Gunnar Johansson ${ }^{1, *}$, Sheila Bingham ${ }^{2}$ and Marie Vahter ${ }^{3}$ \\ 'Department of Food and Nutrition, University of Umeå, SE-901 87 Umeå, Sweden: ${ }^{2}$ MRC Dunn Clinical \\ Nutrition Centre, Hills Road, Cambridge CB2 2DH, UK: ${ }^{3}$ Institute of Environmental Medicine, Karolinska Institute, \\ Box 210, SE-171 77 Stockholm, Sweden
}

Submitted: 1 February 1999: Accepted 15 June 1999

\begin{abstract}
Objective: To develop a method to make use of incomplete 24-hour urinary samples in nutritional epidemiology, especially when validating the dietary intake of nitrogen (protein), sodium and potassium.

Design: Urinary data for men and women collected in three different studies were evaluated. The concentration of para-aminobenzoic acid (PABA) in one 24-hour urine sample per person was compared with the concentrations of nitrogen, sodium, potassium and creatinine.

Setting: Men and women living in Cambridge, UK and women living in the town of Varberg, Sweden.

Subjects: In total, this study consists of data from 73 Swedish women (20-50 years of age), 165 UK women (50-65 years) and 75 UK men ( $55-88$ years).

Results: On average four out of 10 people in this study had a PABA recovery below $85 \%$. The linear regression equations for urinary excretion of nitrogen, sodium and potassium in relation to PABA recovery were $y=2.3+0.088 * x(r=0.99), y=45+$ $0.82 * x(r=0.87)$ and $y=19+0.60 * x(r=0.93)$, respectively.

Conclusions: The linear regression equations can be used for adjusting urinary nitrogen, sodium and potassium in urinary collections in cases where the PABA recovery is below $85 \%$. Since it is common to obtain 24 -hour urine collections with a PABA recovery below $85 \%$, this method should increase the usefulness of biological markers of food intake in nutritional epidemiological studies and also increase the possibilities to study people that previously have been part of the drop-out group or the group with low motivation and cooperation. It is important to stress that we have not studied the relationship between PABA recovery and various urinary variables below the PABA recovery of $50 \%$. Thus, in a case of PABA recovery below $50 \%$, we do not recommend the use of this method to compensate for incomplete collections.
\end{abstract}

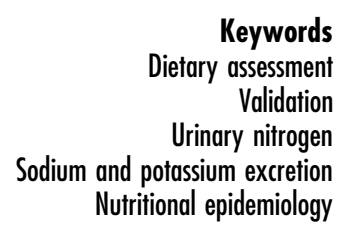

Twenty-four-hour urine collections are often required in clinical investigations, metabolic balance studies and for research purposes. For instance, they are used to assess renal function, to measure exposure to various compounds, to assess sodium intake in nutritional epidemiological studies, and to validate the accuracy of dietary surveys ${ }^{1}$. Incomplete urine sampling may lead to serious errors in the study results. For example, a validation with biological markers based on incomplete urine collections will result in a falsely high ratio of dietary intake/biological marker, since the denominator (the biological marker) will be erroneously low ${ }^{2}$. This will mask underreporting of dietary intakes. Presenting data based on 24-hour urinary collections without verification of their completeness may, therefore, result in incorrect results regarding the validity of the dietary assessment methods, daily excretion of certain compounds, dietary balance studies, etc. and, thus, incorrect conclusions will be drawn.

Para-aminobenzoic acid has been used to check the completeness of urinary collections ${ }^{3,4}$. One tablet of $80 \mathrm{mg}$ PABA should be taken three times on the day of the urine collection: with the morning meal, the midday meal and the evening meal. It is relatively easy to check if the tablets have been taken (if there are any tablets left at the end of the day), when they have been taken (written down in a protocol), when meals have been taken, or if the person has a disease that may influence the excretion rate. However, it may be difficult to remember if any urine specimens have been omitted from the 24-hour collection. The PABA is assumed to be excreted almost quantitatively within 
24 hours, and 24-hour collections containing less than $85 \%{ }^{3}$ or less than $78 \%{ }^{4}$ of the ingested PABA marker have been classified as incomplete.

Jakobsen et al. ${ }^{4}$ have improved the PABA method by replacing the colorimetric method with a highperformance liquid chromatography (HPLC) method. With this, the interference from different drugs, including paracetamol, is eliminated. The lowest acceptable recovery of PABA for assessing completeness of 24hour urinary collections is $78 \%(187 \mathrm{mg} / 24$ hours $)$ in a single observation when $240 \mathrm{mg}$ (three times $80 \mathrm{mg}$ ) of PABA is taken.

Usually 24-hour urinary collections that are known to be incomplete are discarded. A method to compensate for incomplete urine collections would therefore be of great practical and economical value. The aim of the study was to:

1. Establish the prevalence of urinary samples with PABA recoveries below $85 \%$ in studies we have been involved in.

2. Illustrate the consequences of using urine samples with a PABA recovery below $85 \%$.

3. Develop a method that can make use of 24-hour urinary samples with a PABA recovery below $85 \%$ in any study where the daily urinary excretion is measured, especially in nutritional epidemiology when validating the dietary intake of nitrogen (protein), sodium and potassium.

\section{Material and methods}

This study is based on data from three previous studies: 73 Swedish women aged $20-50$ years ${ }^{5}, 165$ UK women aged 50-65 years ${ }^{6}$ and 75 UK men aged $55-88$ years $^{7}$ (and the authors' unpublished data). In the UK studies eight 24-hour urine samples per person were collected and in the Swedish study one 24-hour urine sample per person was collected. Subjects who had no verified problems in collecting a 24-hour urinary sample, who were without any medication that could interfere with the PABA test and who had a PABA recovery between 50\% and $110 \%$ were included in the study.

The subjects had been asked to record the start (after the first morning urine) and the end (right after the morning urine the next day) of the 24-hour urine collection period, and to report any lost specimens. They were given three $80 \mathrm{mg}$ tablets of PABA (supplied by the Laboratories for Applied Biology, London, UK and the Karolinska Hospital, Stockholm, Sweden) to take with morning, mid-day and evening meals. PABA in the urine was measured colorimetrically ${ }^{3}$. Total urinary nitrogen was determined by the Kjeldahl technique (Tecator 1002, Perstorp Analytical, Bristol) ${ }^{1}$. Urinary sodium and potassium were determined by flame atomic absorption spectrophotometry. Creatinine in the urine was analysed according to Hare ${ }^{8}$.

The urinary excretion of nitrogen, sodium and potassium was evaluated against PABA recovery using linear regression analysis. Data for one 24-hour urine sample per person was used (the first collection, if more than one was collected) and the PABA recovery values were divided into 5\% intervals from 50\% to $90 \%$ and one interval between $90 \%$ and $110 \%$. We have in this study, compensated values under $85 \%$ up to $93 \%$ as Bingham and Cummings ${ }^{3}$ found in their urinary completeness validation study, a mean PABA recovery of $93 \pm 4 \%(n=33)$. This is made by using the linear regression equation:

$$
y=a+\beta * x,
$$

where $y$ is the analyte in urine, in this case nitrogen, sodium or potassium, $a$ is the analyte in urine when the PABA recovery is zero, $\beta$ is the slope of the curve and $x$ is the PABA recovery value (93\% minus the measured value).

For instance, if a urine sample had a PABA recovery of $63 \%$ and a nitrogen content of $14.5 \mathrm{~g}$, the linear regression equation $(\beta * x)$ will give $0.088 *(93-63)=$ $2.6 \mathrm{~g}$. Thus, the adjusted nitrogen output would be $14.5+2.6=17.1 \mathrm{~g}$. The figure 0.088 for the slope of the curve is a result of the present study.

\section{Results}

In total, 39\% of the subjects collecting 24-hour urine samples had a PABA recovery below $85 \%$. In the UK study on men, 227 out of a total of 582 collections (39\%) had a PABA recovery below $85 \%$. Twelve men (16\%) had all eight 24-hour urine samples with a PABA recovery over $84 \%$ and five men (7\%) did not have any of the eight collections with a PABA recovery over $84 \%$. In the first collection of the UK study on women, 44 out of 165 women (27\%) had a PABA recovery below $85 \%$. In the Swedish study, 52 out of 73 women $(71 \%)$ had a PABA recovery below $85 \%$.

The ratio of the dietary intake/biological marker in relation to PABA recovery in the Swedish study ${ }^{9}$ shows that the lowest PABA recovery group (PABA $<70 \%$; mean 55\%) had the highest ratio values, and that the ratio values for the group with a mean PABA recovery of $90 \%$ (PABA $>85 \%)$ were similar to those of the 'adjusted group' (Table 1). The adjustment is performed according to the linear regression method presented in this study.

The linear regression equations for urinary nitrogen, sodium and potassium in relation to PABA recovery based on data from all three studies were:

- Urinary nitrogen: $y=2.3+0.088 * x, r=0.99$ (Fig. 1).

- Sodium: $y=45+0.82 * x, r=0.87$ (Fig. 2).

- Potassium: $y=19+0.60 * x, r=0.93$ (Fig. 3). 
Table 1 The ratio of dietary intake according to the food record method or duplicate diet technique to dietary intake according to the biological marker is presented in relation to the PABA recovery. The first $(55 \%)$ and second $(90 \%)$ groups are the total group of women in the Swedish study divided into two PABA recovery groups (less than $70 \%$ and over $85 \%$ ). The third group is the total number of women adjusted for PABA recoveries under $85 \%$ up to $93 \%$ as described in this study

\begin{tabular}{lccc}
\hline & \multicolumn{3}{c}{ Mean PABA recovery value } \\
\cline { 2 - 4 } Variable & $\begin{array}{c}55 \% \\
(n=28)\end{array}$ & $\begin{array}{c}90 \% \\
(n=21)\end{array}$ & $\begin{array}{c}\text { Adjusted } \\
(n=73)\end{array}$ \\
\hline Protein* & 1.78 & 0.91 & 0.86 \\
Protein $\dagger$ & 1.14 & 0.90 & 0.85 \\
Sodium $\ddagger$ & 1.19 & 0.88 & 0.84 \\
Potassium§ & 1.15 & 0.87 & 0.82 \\
\hline
\end{tabular}

* The protein intake according to the food record/protein intake according to urinary nitrogen, $6.25^{*}$ (nitrogen in urine +2 ).

$\dagger$ The protein intake according to the duplicate diet/protein intake according to urinary nitrogen, $6.25^{*}$ (nitrogen in urine +2 ).

$\ddagger$ The sodium intake according to the food record/sodium in urine.

$\S$ The potassium intake according to the food record/potassium in urine and faeces.

The mean values and standard deviations for the data presented in Figs 1-3 is shown in Table 2.

When the urinary variables creatinine, volume, nitrogen, sodium and potassium from the Swedish study were expressed in relative terms and related to three PABA recovery levels, 55\%, 79\% and 90\%, the linear regression equation was: $y=32+0.75 * x, r=0.99$ (Fig. 4). The relationship for an ideal marker for completeness of urinary samples would be $y=x$, and is included in Fig. 4.

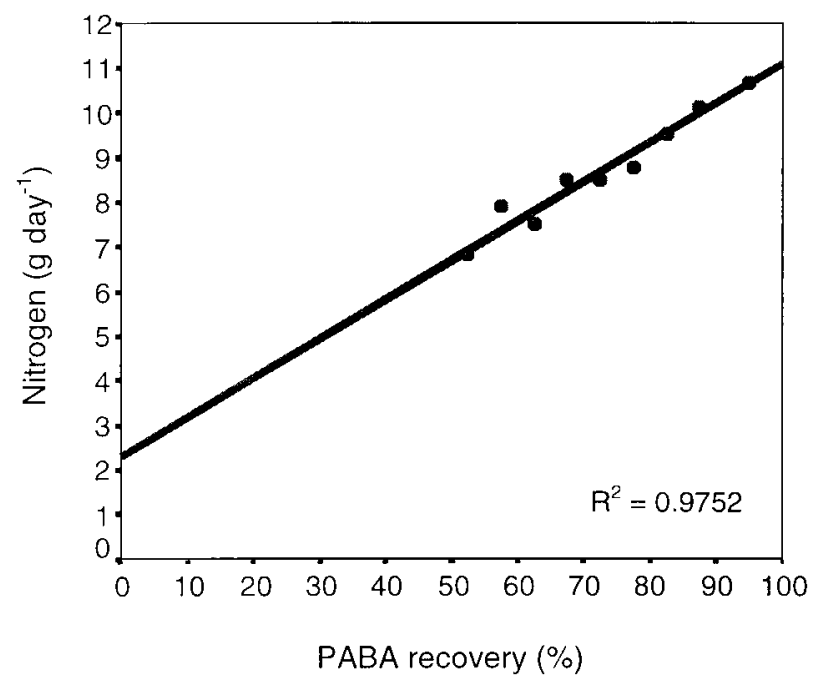

Fig. 1 The relationship between PABA recovery (\%) and nitrogen output in urine $\left(\mathrm{g} \mathrm{day}^{-1}\right)$. The PABA recovery values have been divided into $5 \%$ intervals from $50 \%$ to $90 \%$ and one interval between $90 \%$ and $110 \%$. The number of individuals for each PABA interval is $10,12,15,13,12,19,37,63$ and 131, respectively (total, $n=312$ ). The correlation coefficient $r$ is 0.99 when a weighted calculation is performed, i.e. all 312 values are taken into account in the regression analysis

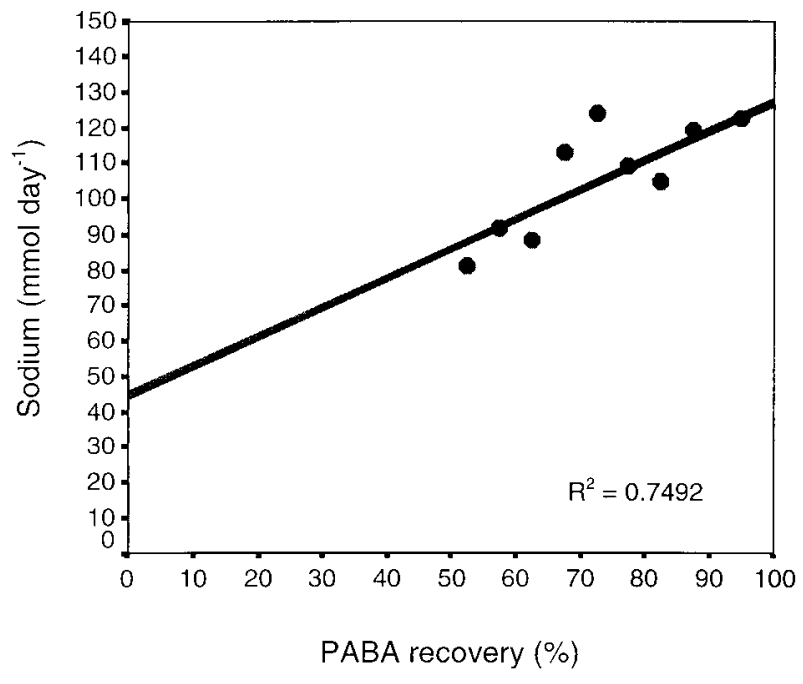

Fig. 2 The relationship between PABA recovery (\%) and sodium output in urine $\left(\mathrm{mmol}^{-1} \mathrm{day}^{-1}\right)$. The PABA recovery values have been divided into $5 \%$ intervals from $50 \%$ to $90 \%$ and one interval between $90 \%$ and $110 \%$. The number of individuals for each PABA interval is $10,12,15,13,12,20,37,63$ and 131 , respectively (total, $n=313$ ). The correlation coefficient $r$ is 0.87 when a weighted calculation is performed, i.e. all 313 values are taken into account in the regression analysis

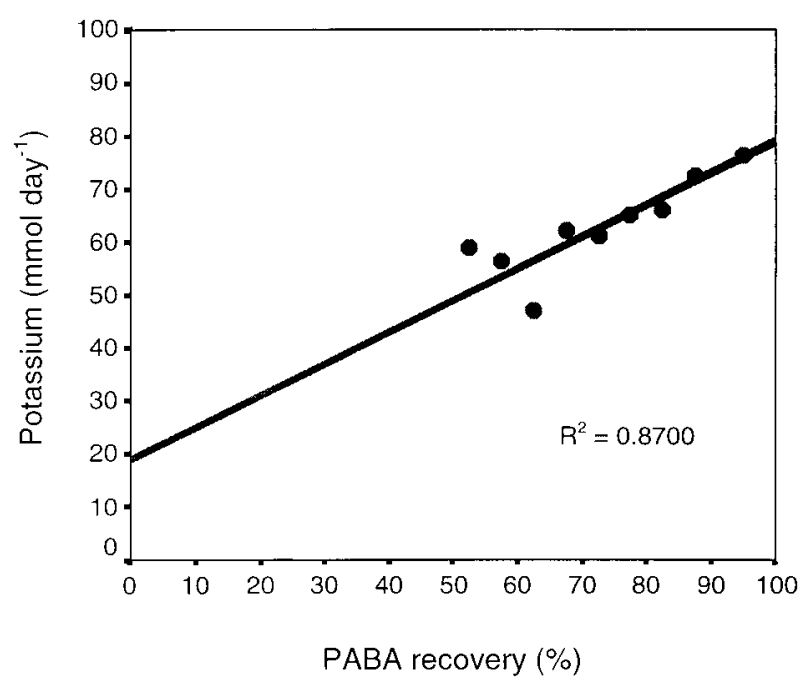

Fig. 3 The relationship between PABA recovery (\%) and potassium output in urine $\left(\mathrm{mmol} \mathrm{day}^{-1}\right)$. The PABA recovery values have been divided into $5 \%$ intervals from $50 \%$ to $90 \%$ and one interval between $90 \%$ and $110 \%$. The number of individuals for each PABA interval is $10,12,15,13,12,20,37,63$ and 131 , respectively (total, $n=313$ ). The correlation coefficient $r$ is 0.93 when a weighted calculation is performed, i.e. all 313 values are taken into account in the regression analysis

\section{Discussion}

The PABA method is presently the only available test to check the completeness of urine collections. Even though Jakobsen et $a l^{4}{ }^{4}$ solved the interference problem of some widely used drugs, especially paracetamol and sulphonamides, the PABA test has 
Table 2 The mean values and standard deviations (in brackets) for each PABA interval for the data presented on nitrogen, sodium and potassium in urine in Figs $1-3$. The PABA recovery values have been divided into $5 \%$ intervals from $50 \%$ to $90 \%$ and one interval between $90 \%$ and $110 \%$. The total $n$ value is 312 for nitrogen and 313 for sodium and potassium

\begin{tabular}{lcrrr}
\hline $\begin{array}{l}\text { PABA } \\
\text { recovery value (\%) }\end{array}$ & $n$ & Nitrogen & Sodium & Potassium \\
\hline $50-54$ & 10 & $6.8(2.2)$ & $81(23)$ & $59(21)$ \\
$55-59$ & 12 & $7.9(2.7)$ & $92(42)$ & $56(22)$ \\
$60-64$ & 15 & $7.5(1.8)$ & $88(20)$ & $47(11)$ \\
$65-69$ & 13 & $8.5(3.1)$ & $113(39)$ & $62(21)$ \\
$70-74$ & 12 & $8.5(1.6)$ & $123(44)$ & $61(11)$ \\
$75-79$ & 20 & $8.8(1.8)^{\star}$ & $109(43)$ & $65(21)$ \\
$80-84$ & 37 & $9.5(2.3)$ & $104(44)$ & $66(26)$ \\
$85-89$ & 63 & $10.1(2.3)$ & $119(40)$ & $72(18)$ \\
$90-110$ & 131 & $10.7(2.5)$ & $123(45)$ & $76(20)$ \\
\hline${ }^{*} n=19$ & & & &
\end{tabular}

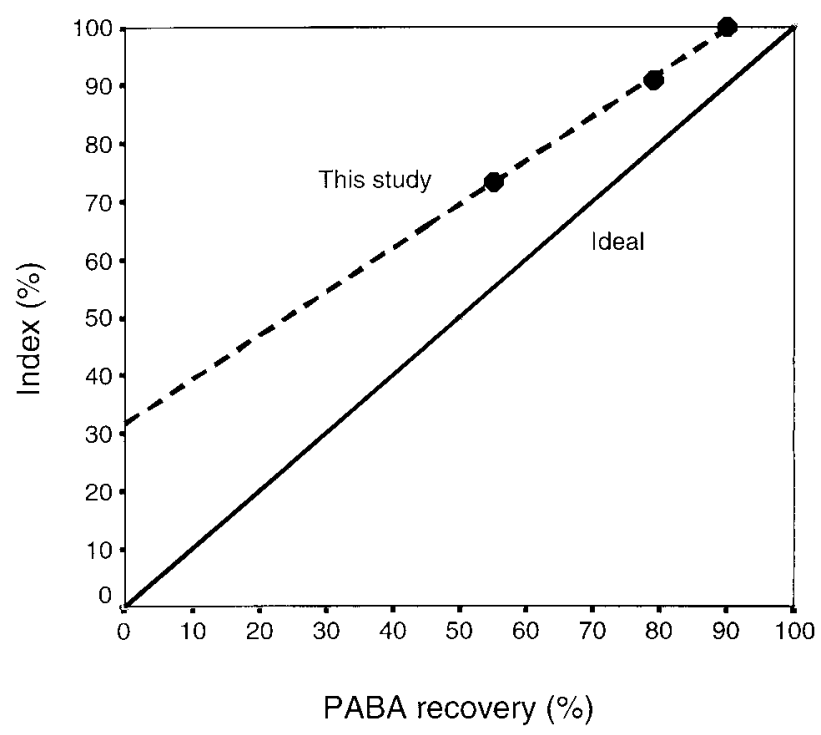

Fig. 4 The relationship between the PABA recovery and the mean values for urinary creatinine, volume, protein (derived from nitrogen), sodium and potassium for three groups in the Swedish study with a mean PABA recovery of $55 \%, 79 \%$ and $90 \%$, respectively. The urinary variables are expressed in relative terms, where the urinary values at $90 \%$ PABA recovery is set to 1.00 and the values for the urinary variables at $55 \%$ and $79 \%$ are expressed in relation to the $90 \%$ value. The correlation coefficient $r$ is 0.99 . The ideal relationship for an urinary marker is added $(y=x)$

not been fully evaluated. For instance, variation with age, analytical variation, interactions with various foods and the biological variation within and between individuals on a large scale have not been fully investigated.

In the three studies reviewed, approximately four out of 10 people had a PABA recovery below $85 \%$. We have similar experiences from other studies we have been involved in. Also, other researchers in this area confirm that our findings are not rare (E Rothenberg, personal communication). Although all the reasons for low PABA recoveries are not known, the results indicate serious problems in collecting 24-hour urinary samples and, thus, in the measurements of daily urinary excretion of various compounds, e.g. in studies of human exposure of chemicals and in validating dietary surveys.

The present study showed that the measured 24-hour urinary excretion of nitrogen, sodium and potassium increased with increasing urinary PABA recovery. This indicates that, at least on a group level, a low PABA recovery is related to incomplete urine collections. The fact that the slope of the linear regression curves for the empirical relationships found in this study did not intercept origo and the linear regression equations were not $y=x$ (Figs 1-4) - as it would be if PABA was a perfect marker for detecting incomplete urinary samples - indicate that there are other reasons than undercollection that may cause low PABA recovery values. Such reasons may include:

1. One or more tablets were not taken ${ }^{1-4,8,10,11}$.

2. Tablets were taken late in the evening, especially together with a (large) meal that slows down the emptying of the stomach and the uptake in the intestines ${ }^{10}$.

3. Biological variation in PABA pharmacokinetics.

4. Analytical errors?.

5. Renal detoriation or failure $e^{11,12}$

6. Errors in the preparation of the aliquots?

These items need more research.

It is important to stress that we have not studied the relationship between PABA recovery and various urinary variables below the PABA recovery level of $50 \%$. Thus, in cases of PABA recoveries below 50\%, we do not recommend the use of this method to compensate for incomplete collections.

\section{Conclusions}

We have presented a method to adjust 24-hour urine collections with a PABA recovery between $50 \%$ and $85 \%$ (the colorimetric method), up to $93 \%$. It is based on the statistical relationship between the PABA recovery and the amount of analytes in the urine. By this method, the errors introduced by incomplete urine collections will most likely be reduced, although not completely eliminated. This method should increase the usefulness of biological markers of food intake based on 24-hour urinary samples in nutritional epidemiological studies by increasing the possibilities of studying people that previously have been part of the drop-out group or the group with low motivation and cooperation (i.e. that were poor urinary collectors). Even though this method could improve some studies, reduce the drop-out rate in some studies and make some studies possible, it should not deter us from searching for a more specific marker for measuring the completeness of urine collections.

Although the procedure reported here does allow 
correction for incomplete recovery, it cannot be used as a substitute for a careful approach which includes clear and detailed instructions to participants who are asked to make a complete 24-hour urine collection.

\section{Acknowledgements}

We thank Shirley Runswick, Agneta Åkesson, Barbro Nermell and Marika Berglund for carrying out skilful and careful work concerning laboratory analyses and fieldwork.

\section{References}

1 Bingham S, Cummings JH. Urine nitrogen as an independent validatory measure of dietary intake: a study of nitrogen balance in individuals consuming their normal diet. Am.J. Clin. Nutr. 1985; 42: 1276-89.

2 Bingham SA. The use of 24-h urine samples and energy expenditure to validate dietary assessments. Am. J. Clin. Nutr. 1994; 59: S227-31.

3 Bingham S, Cummings JH. The use of 4-aminobenzoic acid as a marker to validate the completeness of $24 \mathrm{~h}$ urine collections in man. Clin. Sci. 1983; 64: 629-35.

4 Jakobsen J, Ovesen L, Fagt S, Pedersen AN. Para-aminobenzoic acid used as a marker for completeness of 24 hour urine: assessment of control limits for a specific HPLC method. Eur. J. Clin. Nutr. 1997; 51: 514-19.
5 Vahter M, Berglund M, Nermell B, Åkesson A. Bioavailability of cadmium from shellfish and mixed diet in women. Toxicol. Appl. Pharmacol. 1996; 136: 332-41.

6 Bingham SA, Gill C, Welch A, et al. Comparison of dietary assessment methods in nutritional epidemiology: weighed records v. 24-h recalls, food-frequency questionnaires and estimated-diet records. Br. J. Nutr. 1994; 72: 619-43.

7 Black AE, Bingham SA, Johansson G, Coward WA. Validation of dietary intakes of protein and energy against 24 hour urinary $\mathrm{N}$ and DLW energy expenditure in middle-aged women, retired men and post-obese subjects: comparison with validation against presumed energy requirements. Eur. J. Clin. Nutr. 1997; 51: 405-13.

8 Hare R. Endogenous creatinine serum and urine. Proc. Soc. Exp. Biol. Med. 1950; 74: 148-51.

9 Johansson G, Åkesson A, Berglund M, Nermell B, Vahter M. Validation with biological markers for food intake of a dietary assessment method used by Swedish women with three different dietary preferences. Public Health Nutr. 1998; 1(3): 199-206.

10 Knuiman JT, Hautvast JGAJ, Van der Heyden L, et al. A multicentre study on completeness of urine collection in 11 European centres. Hum. Nutr. Clin. Nutr. 1986; 40C: 22937.

11 Bingham SA, Murphy J, Waller E, et al. Para-amino benzoic acid in the assessment of completeness of 24-hour urine collections from hospital outpatients and the effect of impaired renal function. Eur. J. Clin. Nutr. 1992; 46: 131-5.

12 Papper S. The effect of age in reducing renal function. Geriatrics 1973; 28(5): 83-7. 